\title{
Subwavelength Structured Surfaces and their Applications
}

\author{
Daniel H. Raguin and G. Michael Morris \\ The Institute of Optics, University of Rochester, Rochester, NY 14627, (716) 275-8008
}

\section{Introduction}

The term subwavelength structured (SWS) surface describes any surface that contains a subwavelength-period grating or gratings, see Fig. 1. The grating may be of any type (e.g., surface-relief, phase, or amplitude) provided the period is sufficiently fine so that, unlike conventional gratings, no diffraction orders propagate other than the zeroth orders. Because of the fine periods involved, the fabrication of such surfaces for applications in the visible and infrared (IR) portions of spectral regime have only recently been considered. With refinements in holographic procedures and the push of the semiconductor industry for submicron lithography (both through optical and electron beam techniques), production of SWS surfaces is becoming increasingly viable.

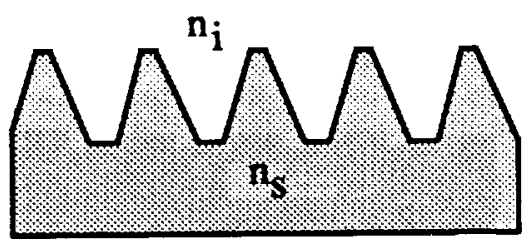

(a)

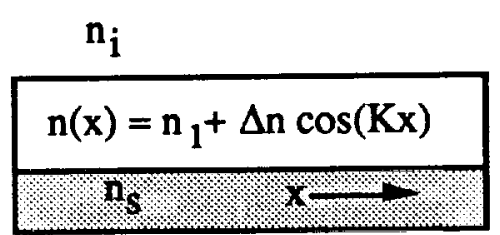

(b)

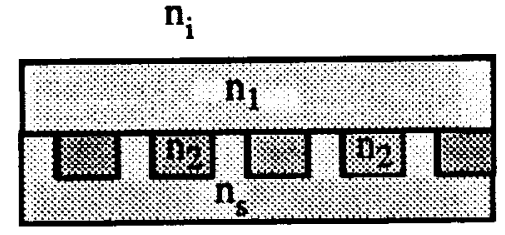

(c)

Fig. 1. Possible SWS surface geometries: (a) surface-relief profile, (b) phase grating, and (c) embedded surface-relief profile.

There are numerous applications for SWS surfaces: antireflection coatings, polarization components, narrow-band filters, and phase plates. As antireflection components, SWS surfaces are called antireflection structured (ARS) surfaces and have been studied extensively both theoretically and experimentally (see, for example, Refs. 1-7). Typically, ARS surfaces contain surface-relief gratings, see Fig. 1(a), which impedance match two media where one is a solid, and the other a gas, liquid, or solid. By structuring a surface with a subwavelength-period pattern (either through etching, embossing, or other techniques), one can synthesize an appropriate index of refraction distribution so that surface reflections are minimized. When designed properly, these structures can operate over large spectral bandwidths and fields of 
view. ${ }^{6}$ Because foreign materials are not being added to the substrate surface, problems commonly encountered in thin-film technology, such as cohesion and thermal expansion mismatches, are non-existent in the design of these structured surfaces. Although relatively new to mankind, ARS surfaces can be found on the cornea of certain night-flying moths. ${ }^{8}$ The subwavelength structures of the moth's cornea reduce surface reflections which would otherwise betray the moth's position to its predators. The first scientists to investigate ARS surfaces for application in the visible or near-IR portion of the spectrum worked to replicate moth-eye surfaces (see, for example, Ref. 1). Only later were non-moth-eye profiles investigated. ${ }^{2-7}$

As polarization components, SWS surfaces may take any form diagrammed in Fig. 1. These surfaces operate on the fact that unlike in the scalar diffraction regime (where the surface period is large compared to the incident wavelength), when feature sizes are on the order of a wavelength, then, in general, radiation will react to the surface differently depending on the electric field's polarization orientation. SWS surfaces may be used to fabricate wire grid polarizers, ${ }^{9}$ beam splitters, ${ }^{10}$ waveplates and retarders, ${ }^{11,12}$ and polarizing mirrors. ${ }^{13}$ SWS surfaces may be applied to any portion of the electromagnetic spectrum while conventional polarization devices must rely on the existence of a suitable birefringent material for the particular wavelength range of interest. Since the magnitude of the effective birefringence $\Delta \boldsymbol{n}$ (equal to $n_{e}-n_{o}$, where $n_{e}$ and $n_{o}$ are the structure's effective extraordinary and ordinary indices of refraction, respectively) can be quite large (e.g., for fused silica $\Delta n \approx-0.1$, for $\mathrm{ZnSe}$ $\Delta n \approx-0.65$, and for GaAs $\Delta n \approx-1.7$ ), the grating of a SWS surface need not be deeper than the wavelength of the incident radiation. Consequently, SWS surfaces are less bulky than conventional polarization devices and offer the possibility of replacing several conventional elements with a single structured one (e.g., beamsplitter waveplates or mirror waveplates).

A typical geometry of SWS surfaces for use as narrow-band filters is illustrated in Fig. 1(c). By filling the grooves of the grating with a material of index $n_{2}$ where $n_{2}$ is larger than $n_{1}$ or $n_{S}$, the structure can support the propagation of leaky waveguide modes. Coupling between the incident field and these leaky modes results in extremely sharp changes in the structure's spectral characteristics as a function of angle of incidence and wavelength. It is due to the highly selective coupling criterion of the waveguide mode that these structures can exhibit extremely narrow filter linewidths $\left(\Delta \lambda / \Lambda=10^{-7}\right) .{ }^{14}$ Applications employing narrow-band filters are numerous. One field which can benefit tremendously from this new technology is the laser field. Laser end mirrors may be fabricated to reflect $100 \%$ of the incident radiation for a fixed wavelength. Since the narrow-band filter effects are polarization dependent, a laser with a SWS end mirror does not need any Brewster windows. Since the reflection spectra of these 
SWS surfaces change remarkably as a function of incident angle, M. T. Gale has suggested utilizing these devices for security applications much in the same way that holograms are used on credit cards today. ${ }^{15}$

SWS surfaces may also be used as an alternative method of fabricating phase plates. ${ }^{16,17}$ By fabricating a binary SWS surfaces in which the duty cycle of the profile varies across the surface, any desired phasefront may be imparted onto the incoming radiation. Although the physical profile depth encountered by the radiation is the same at all points across the surface, due to the surface's changing filling factor, the optical path length accounted by the radiation will vary from point to point. By using a binary profile with subwavelength features, efficiencies may still be high (unlike binary profiles in the scalar regime) without resorting to a multi-mask fabrication process.

\section{Analytic Approaches}

To analyze SWS surfaces, the use of vector diffraction theory is required since due to the feature sizes involved, scalar diffraction theories are completely invalid. Both coupled-wave and modal approaches are valid (and equivalent) techniques for modeling radiation's interaction with SWS surfaces. ${ }^{18}$ The vector analysis performed for the research presented takes advantage of the rigorous coupled-wave analysis (RCWA) proposed by Gaylord and Moharam. ${ }^{18,} 19$

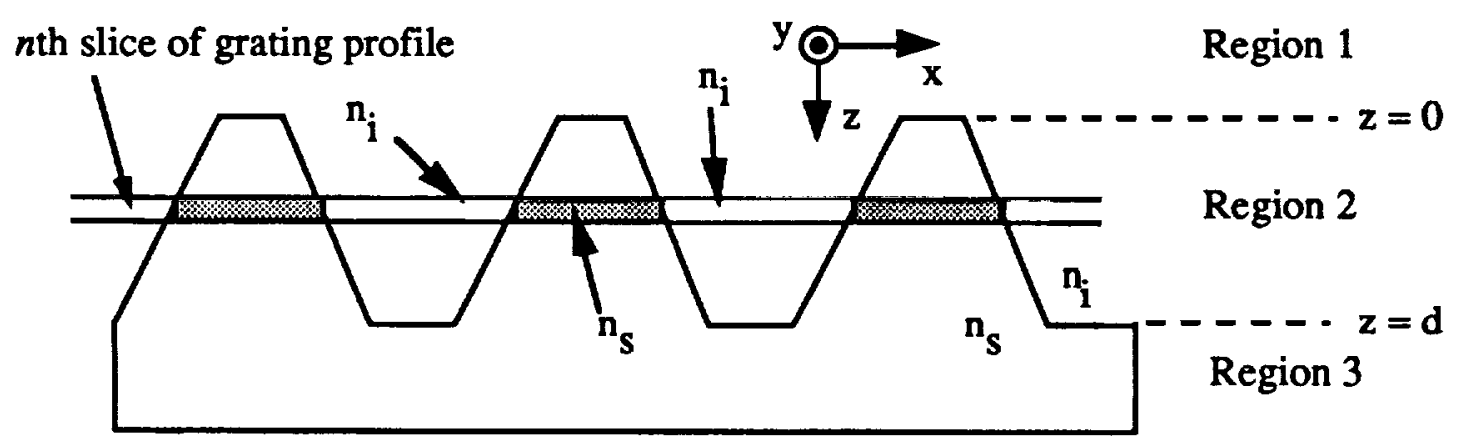

Fig. 2. Grating geometry. In rigorous coupled-wave analysis (RCWA), a grating with a continuous profile is approximated by an $N$-level profile where each layer or slice is analyzed as a thin grating.

RCWA approaches the problem by solving Maxwell's equations without making any assumptions regarding the profile period or depth. Grating profiles which vary as a function of depth, such as the continuous surface-relief profile diagrammed in Fig. 2, are approximated by $N$ slices where in each slice, the optical parameters have no $z$ dependence (a continuous surface- 
relief profile is therefore represented as a multi-level profile). Provided that each slice is sufficiently thin, a grating which varies in $z$ may be accurately modeled. Each slice of the grating region (Region 2 ) is treated as a thin grating and the fields within the slice expanded using the Floquet condition. These fields are substituted into the wave equation, resulting in an infinite set of second-order coupled-differential equations for the field space-harmonic amplitude. This set of equations is reduced to a doubly infinite set of first-order coupled-differential equations using a state-space representation. The space-harmonic amplitudes are extracted in terms of eigenvalues and eigenvectors of the resulting coefficient matrix. Maxwellian boundary conditions are then applied to the harmonic field amplitudes at each layer as well as at the boundaries of the incident medium (Region 1) and the substrate medium (Region 3). Reflection and transmission coefficients are obtained from the amplitudes of the propagating orders in Region 1 and Region 3, respectively.

Like most numerical techniques, RCWA, due to its intensive computational requirements, offers little opportunity for intuitive insight into the diffraction mechanisms. An alternative method for analyzing SWS surfaces is through effective medium theory (EMT). By relying on the fact that the structures have feature sizes smaller than the incident wavelength, an approximate description of the interaction of radiation with such structures can be obtained. The results are analytic and offer a great deal of insight towards the radiation's behavior.

Effective medium theories (EMTs) rely on the fact that when light interacts with periodic structures finer than its wavelength, it does not diffract, but instead reflects and transmits as if it is encountering a non-structured medium. EMTs describe the interaction of light with such subwavelength structures by representing regions of subwavelength heterogeneity in terms of a homogeneous material possessing a single set of effective optical constants: permittivity $\varepsilon$, permeability $\mu$, and conductivity $\sigma$. This is sensible since no energy is lost to diffracted orders. All the energy is contained in the zeroth transmitted and zeroth reflected orders. The optical properties of the effective medium is governed by the specific structural intermixing between the incident and substrate material, but in general, the more substrate material present as compared to incident material in a given region, the closer that region's optical properties are to that of the substrate. In other words, when light interacts with subwavelength structures, it reacts to them as if it were encountering an effective medium whose optical properties are a weighted spatial average of the profile region's optical properties. For the specific case of a multi-level surfacerelief profile, see Fig. 3(a), the effective medium will be a film stack where each layer of the film stack corresponds to a distinct level of the surface-relief profile. For a continuous profile, see Fig. 3(b), the effective medium will be a gradient film. Because the effective medium results from the weighted spatial averaging of the profile region's optical properties, the optical 
properties of the effective medium must be bound by the properties of the incident medium and those of the substrate medium. Therefore, assuming $n_{i}<n_{s}$, in Fig. 3(a), $n_{i}<n_{1}<n_{2}<n_{3}<n_{s}$, and in Fig. 3(b), $n_{i} \leq n(z) \leq n_{s}$.

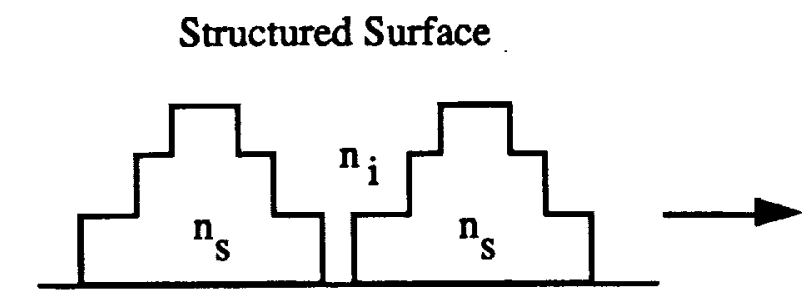

Multi-level Profile

(a)

Effective Medium

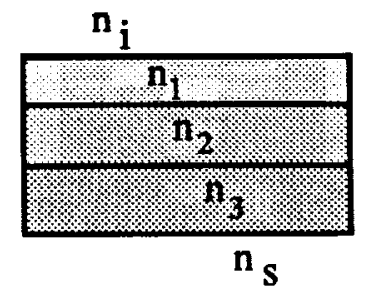

Film Stack

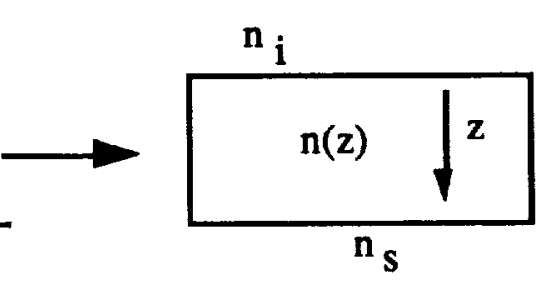

(b) Gradient-Index Film

Fig. 3. Effective mediums: (a) for a multi-level surface profile the effective medium is a film stack, and (b) for a continuous surface profile the effective medium is a gradient-index film.

\section{One-Dimensional Periodic Stratification and Effective Medium Theory}

A one-dimensional (1-D) profile, see Fig. 4, is one of the more common profiles used by optical engineers. This profile may be fabricated through numerous techniques including optical and e-beam lithography, holography, and diamond ruling. Conventionally termed a linear diffraction grating, by making the period fine enough (or the incident wavelength large enough), these structures form one class of SWS surfaces. By determining the effective index of refraction at each depth $z$ into the profile, the effective medium of the grating region is determined. Note that the effective index at a level $z=z_{0}$, is a function of the filling factor $f\left(z_{0}\right)$ at that level, where the filling factor describes the fraction of substrate material present in a period $\Lambda$, see Fig. 4(b). 


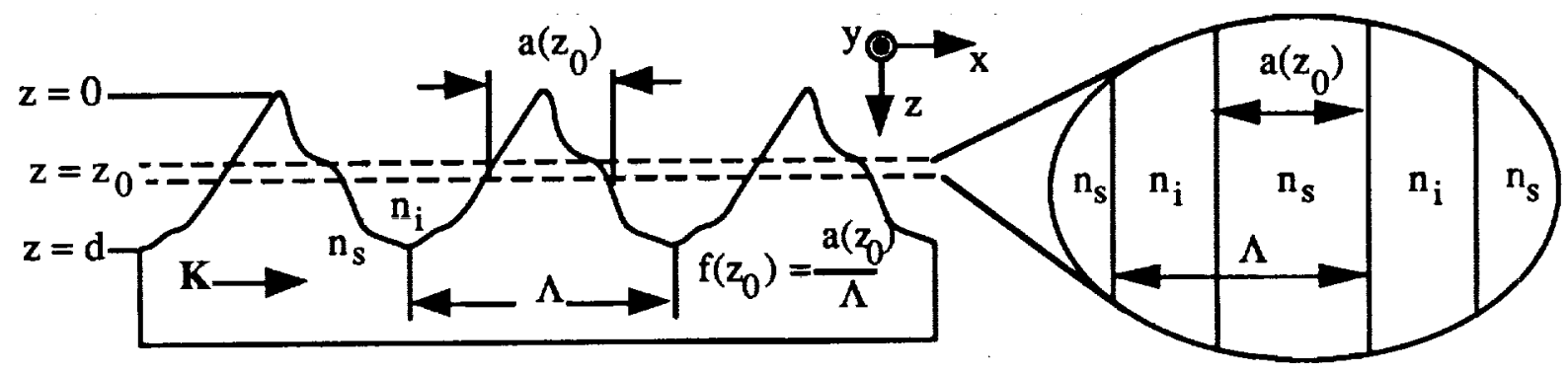

(a)

(b)

Fig. 4. General surface-relief SWS surface. In (a) the profile geometry is diagrammed, while in (b) the stratification present at a depth $z=z_{0}$ is illustrated. The filling factor at a depth $z_{0}$ is equal to $f\left(z_{0}\right)=a\left(z_{0}\right) / \Lambda$.

To describe 1-D ARS surfaces, previous authors have utilized zeroth-order EMT, see for example, Refs. 3 and 4. We have found that for most cases (particularly those in which the profile period is only several times smaller than the incident wavelength and/or in substrate materials whose permittivity is large compared to that of the incident medium's), the use of higher-order EMT produces more accurate results when compared to rigorous vector data. ${ }^{6}$ The inaccuracy of zeroth-order EMT is due to the static approximations made in solving for the electromagnetic fields. These approximations result in expressions for the effective optical properties of a structure being independent of the incident wavelength $\lambda$ and the period of the grating $\Lambda$. With the use of higher-order EMT, the dependence of the effective optical properties on $\lambda$ and $\Lambda$ is not neglected.

The higher-order EMT we use is based upon work by S. M. Rytov. 6, 20, 21 As is the case for zeroth-order EMT, higher order EMT predicts that the effective index of refraction for a 1-D SWS surface is dependent upon the orientation of the electric field $\mathbf{E}$ in relation to the grating vector K. The amount of birefringence, $\Delta n$, a 1-D SWS surface possesses may be defined as

$$
\Delta n=n_{\mathbf{E} \| \mathbf{K}}-n_{\mathbf{E} \perp \mathbf{K}}
$$

\section{Design of Waveplates using Form Birefringence}

As may be noted in Section 3, a 1-D subwavelength stratification behaves as an effectively birefringent medium. Since this birefringence is the result of the form of the intermixing of two materials and not due to any molecular birefringence, this effect is termed form birefringence. Form birefringence thus enables the fabrication of polarization components, 
such as waveplates, using isotropic materials. In Fig. 5 we diagram RCWA data for the spectral response of a $\mathrm{ZnSe}$ quarter-wave plate fabricated using a SWS binary profile.

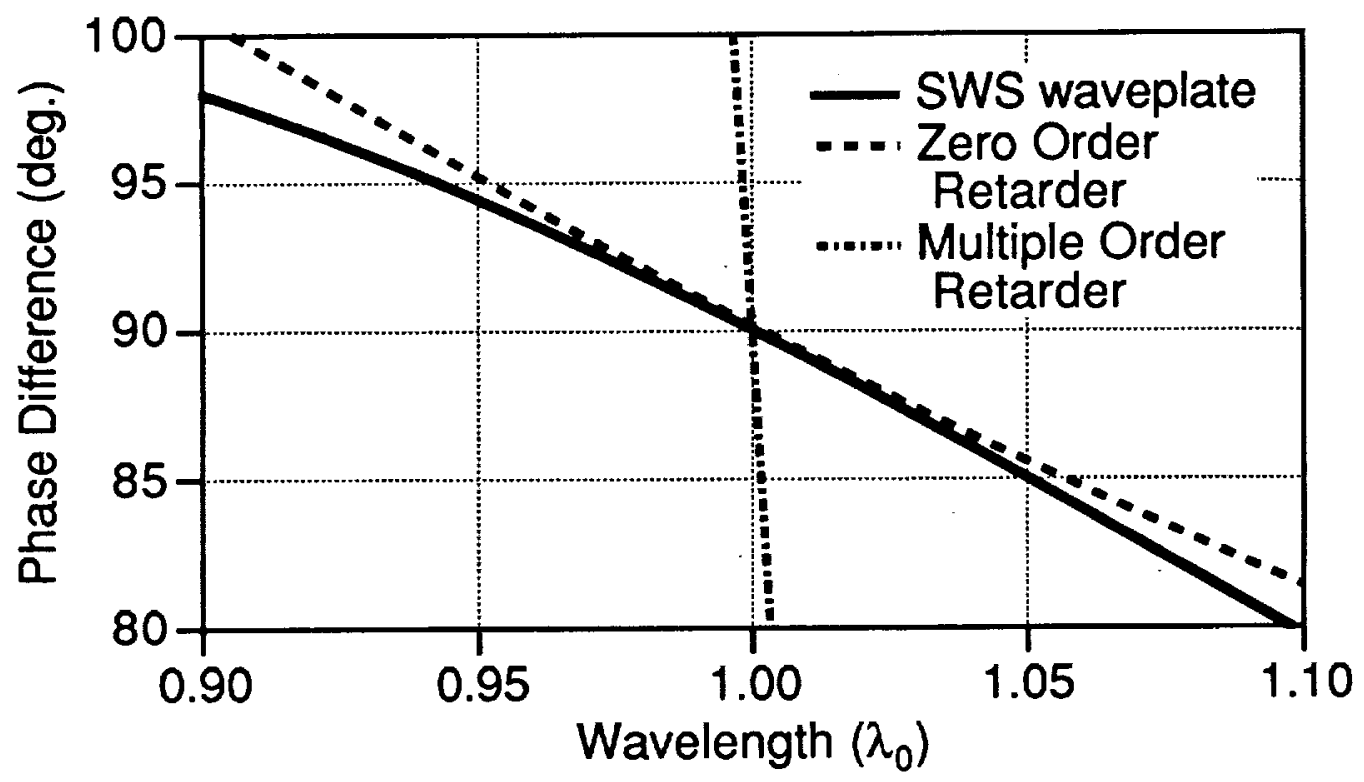

Fig. 5. Spectral performance of a SWS quarter-wave plate as compared to conventional zero-order and multiple-order wave plates.

Due to the large amount of effective birefringence for a $\mathrm{ZnSe}$ binary structure, the binary grating need not be very deep $\left(d=0.455 \lambda_{0}\right)$ to achieve a quarter-wave retardance. For comparisons sake, the wavelength dependence of conventional waveplates, if they existed in the near-IR region of the spectrum, are included. Note that the spectral characteristics of a SWS waveplate is similar to that of a zero-order waveplate. This is not surprising since both devices impart a phase difference of exactly $\Delta \phi=\pi / 2$ on the incident radiation. A multi-order waveplate, on the other hand, imparts a phase difference of $\Delta \phi=\pi / 2+m \pi$ (where $m$ is an integer) and as a result, is highly wavelength sensitive. The period of the $\mathrm{ZnSe}$ waveplate is $\Lambda=0.25 \lambda_{0}$ which means that if the design wavelength $\lambda_{0}$ is scaled, so to must be the period and depth.

A disadvantage with binary profiles as waveplates is the Fresnel losses off the surface. For the case of the $\mathrm{ZnSe}$ waveplate illustrated in Fig. 5, roughly $10 \%$ of the incident radiation is being reflected. One method of reducing the reflection losses is through the use of a continuously profiled surfaces, which will act as an AR coating as well as a waveplate. ${ }^{21} \mathrm{~A}$ continuous profile is able to suppress Fresnel reflections since the optical properties of its effective medium (a gradient film) gradually taper from the properties of the incident medium to 
those of the substrate. Diagrammed in Fig. 6 is a triangular SWS profile along with its effective gradient medium.

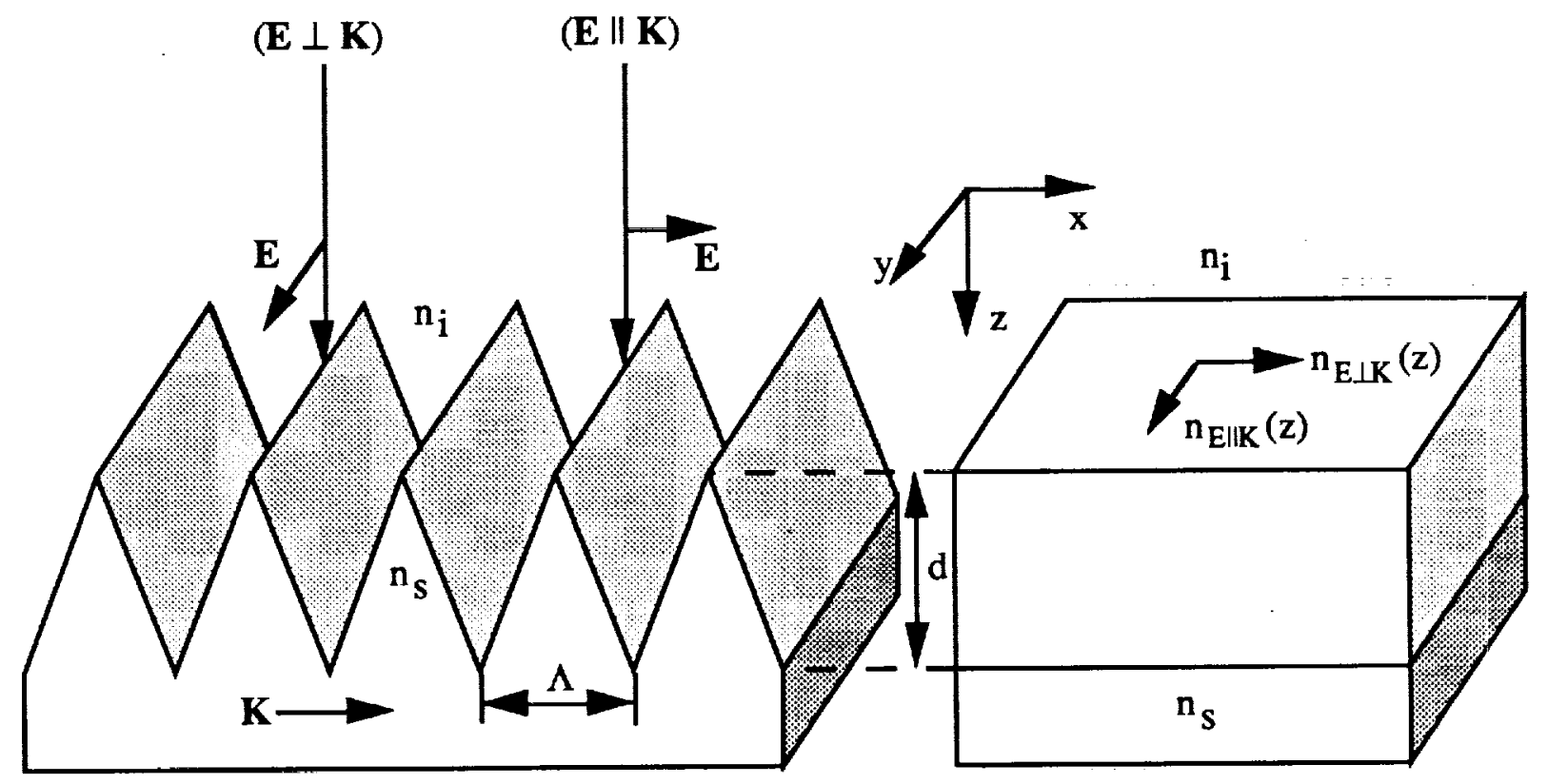

(a)

(b)

Fig. 6. Geometry of (a) a 1-D triangular SWS surface profile along with (b) its effective medium (a gradient film).

Although Fresnel reflections for both polarization states ( $\perp \perp \mathbf{K}$ and $\mathbf{E} \| \mathbf{K}$ ) may be suppressed by 1-D triangular profiles, each polarization state will travel a different optical path length and therefore accumulate a different amount of phase. In Fig. 7 we diagram the phase difference $\Delta \phi$ accumulated by the two orthogonal polarization states as a function of $d$, the depth of the triangular profile. Once the depth of the profile reaches $0.5 \lambda_{0}$, less than $1.5 \%$ of the incident radiation is being reflected from the profile. One may note though that in order to achieve a quarter-wave retardance, the triangular profile must reach a depth of $0.65 \lambda_{0}$ which is deeper than the depth of $0.46 \lambda_{0}$ for the corresponding binary profile. The increase in depth for the triangular profile stems from the fact that the form birefringence of a structure is dependent upon the filling factor $f$, see Eqs. (1)-(6) of the stratification. For a binary profile, the filling factor does not vary with depth, and so can be at or near the filling factor $f_{0}$ which achieves maximum birefringence. For a continuous profile such as the triangular profile diagrammed in Fig. 6, the filling factor, and hence the effective birefringence $\Delta n$, changes with depth. 


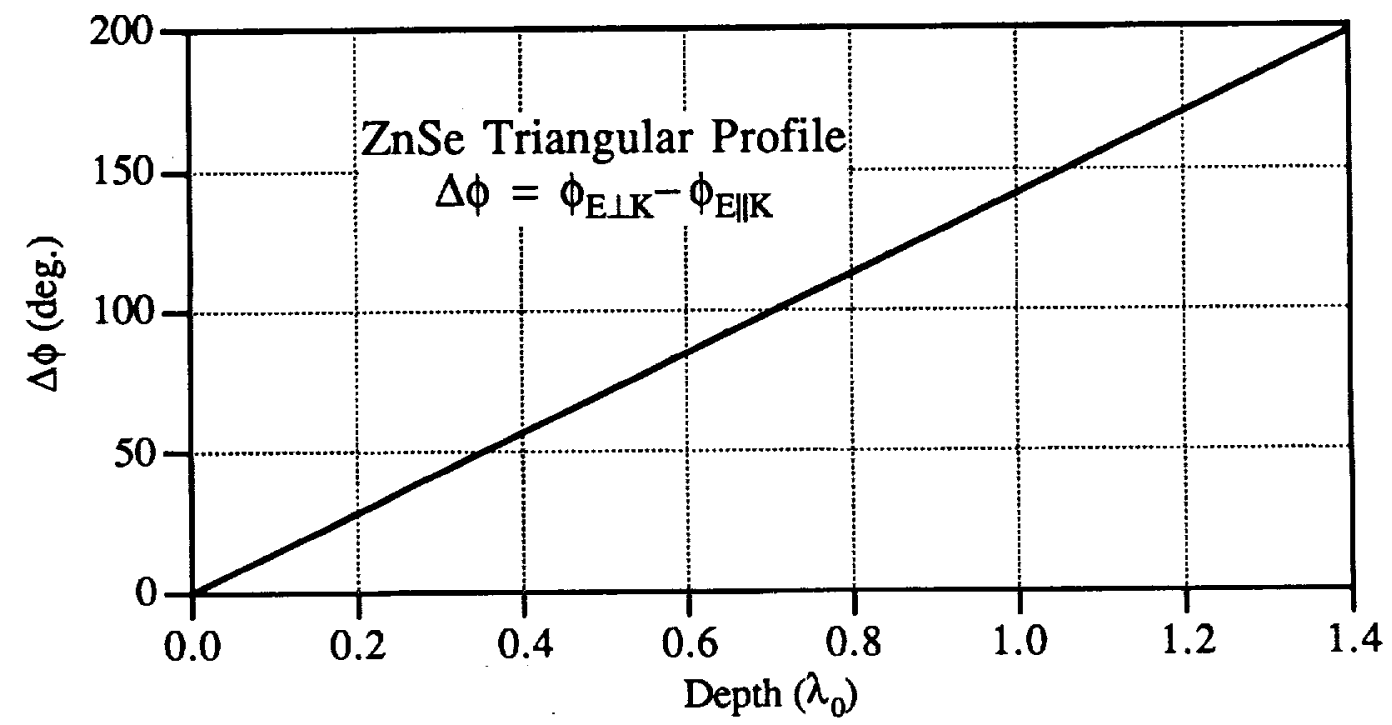

Fig. 7. Phase retardance $\Delta \phi$ accumulated by radiation encountering a triangular SWS profile as a function of profile depth $d$.

\section{2-D Binary Antireflection Structured Surfaces}

For certain applications, birefringent behavior is not desired. One example in which isotropic behavior is preferred is the suppression of a surface's Fresnel reflections for randomlypolarized radiation. Since 1-D SWS surfaces are birefringent due to their surface asymmetry, by increasing the surface symmetry, more isotropic behavior is possible. Near-isotropic behavior may be achieved with a 2-D crossed grating structure.[Raguin, $1992 \# 21$

In Fig. 8 an SEM photograph of a 2-D binary ARS surface is presented. This surface was fabricated on a three-inch silicon wafer using equipment at Cornell's National Nanofabrication Facility (NNF). Since the period of the grating is relatively large $(2.45 \mu \mathrm{m}$ in each direction), we were able to use optical lithography to pattern the substrate. Using optical lithography, the patterning of a three inch wafer takes relatively little time and through step and repeat procedures, larger surfaces may be patterned in under an hour. Etching of the pattern was achieved using reactive ion etching (RIE). These structures are designed to suppress surface reflections for $10.6 \mu \mathrm{m} \mathrm{CO}$ radiation. 


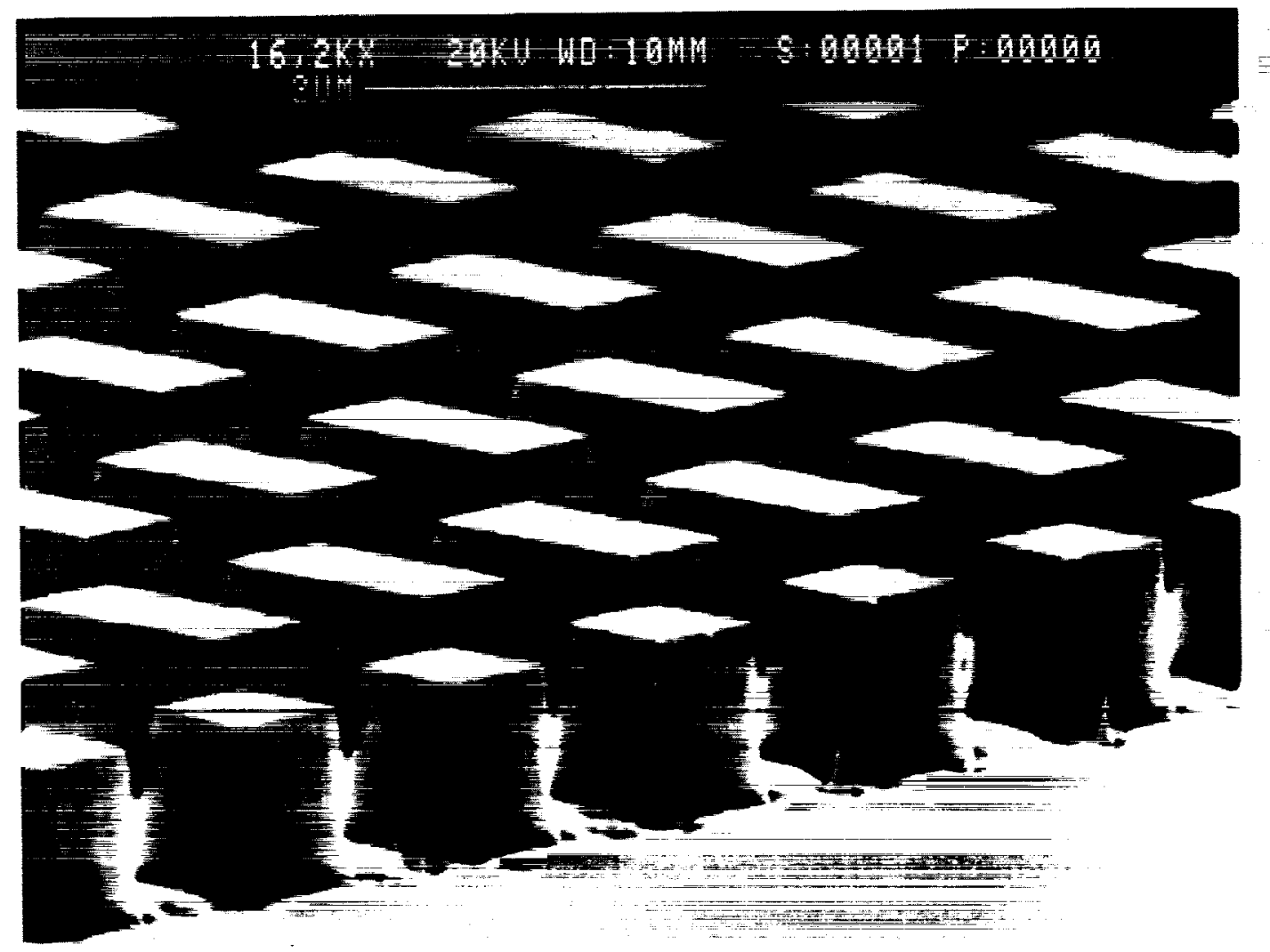

Fig. 8. Silicon 2-D binary ARS surface fabricated on a three-inch wafer.

\section{References}

1. S. J. Wilson and M. C. Hutley, "The optical properties of 'moth eye' antireflection surfaces," Opt. Acta 29, 993-1009 (1982).

2. R. C. Enger and S. K. Case, "Optical elements with ultrahigh spatial-frequency surface corrugations," Appl. Opt. 22, 3220-3228 (1983).

3. T. K. Gaylord, W. E. Baird and M. G. Moharam, "Zero-reflectivity high spatialfrequency rectangular-groove dielectric surface-relief gratings," Appl. Opt. 25, 4562$4567(1986)$.

4. Y. Ono, Y. Kimura, Y. Ohta and N. Nishada, "Antireflection effect in ultrahigh spatialfrequency holographic relief gratings," Appl. Opt. 26, 1142-1146 (1987).

5. N. F. Hartman and T. K. Gaylord, "Anti-reflection gold surface-relief gratings: experimental characteristics," Appl. Opt. 27, 3738-3743 (1988).

6. D. Raguin and G. M. Morris, "Antireflection structured surfaces for the infrared spectral region," accepted for publication in Applied Optics, July 1992. 
7. M. E. Motamedi, W. H. Southwell and W. J. Gunning, "Antireflection surfaces in silicon using binary optics technology," Appl. Opt. 31, 4371-4376 (1992).

8. C. G. Bernhard, "Structural and functional adaptation in a visual system," Endeavor 26, 79-84 (1967).

9. P. Yeh, "A new optical model for wire grid polarizers," Opt. Commun. 26, 289-292 (1978).

10. K. Shiraishi, T. Sato and S. Kawakami, "Experimental verification of a form-birefringent polarization splitter," Appl. Phys. Lett. 58, 211-212 (1991).

11. L. H. Cescato, E. Gluch and N. Streibl, "Holographic quarterwave plates," Appl. Opt. 29, 3286-3290 (1990).

12. C. W. Haggans, L. Li, T. Fujita and R. K. Kostuk, "Lamellar gratings as polarization components for specularly reflected beams," submitted to the Journal of Modern Optics 1992.

13. E. N. Glytsis and T. K. Gaylord, "High-spatial-frequency binary and multilevel stairstep gratings: polarization-selective mirrors and broadband antireflection surfaces," Appl. Opt. 31, 4459-4470 (1992).

14. R. Magnusson and S. S. Wang, "New principle for optical filters," Appl. Phys. Lett. 61, 1022-1024 (1992).

15. M. T. Gale, K. Knop and R. Morf, "Zero-order diffractive microstructures for security applications," Optical Security and Anticounterfeiting Systems, SPIE 1210, pp. 83-89 (1990).

16. M. W. Farn, "Binary gratings with increased efficiency," Appl. Opt. 31, 4453-4458 (1992).

17. H. Haidner, P. Kipfer, W. Stork and N. Steibl, "Zero-order gratings used as an artificial distributed index medium," Optik 89, 107-112 (1992).

18. T. K. Gaylord and M. G. Moharam, "Analysis and applications of optical diffraction by gratings," Proc. IEEE 73, 894-937 (1985).

19. W. B. Veldkamp, G. J. Swanson, S. A. Gaither, C.-L. Chen and T. R. Osborne, "Binary optics: a diffraction analysis," MIT Lincoln Laboratory Project Report ODT 20 (1989).

20. S. M. Rytov, "The electromagnetic properties of finely layered medium," Soviet Phys. JETP 2, 466-475 (1956).

21. D. H. Raguin and G. M. Morris, "Analysis of antireflection structured surfaces with continuous one-dimensional surface profiles," accepted for publication in Applied Optics, Dec. 1992. 
